\title{
Fortalecimiento de las capacidades organizacionales mediante el conversar liberador ${ }^{1}$
}

\author{
Gilda Cecilia Chosco Díaz \\ cdiaz@ungs.edu.ar
}

Jorge Raúl Camblong

jcamblong@ungs.edu.ar
Instituto de Industria, Universidad Nacional de General Sarmiento,

Argentina.
A 100 años de la Reforma Universitaria de 1918 /

Intervenciones
RECEPCIÓN: 29/06/17

ACEPTACIÓN FINAL: 02/10/17

\section{Resumen}

En Argentina, durante las últimas décadas, se desarrollaron experiencias universitarias de fortalecimiento a incubadoras productivas, sociales y tecnológicas. En este sentido, el artículo tiene por objetivo describir una experiencia de fortalecimiento de las "capacidades organizacionales" de las cooperativas de trabajo que conforman el Centro Demostrativo de Indumentaria (CDI). Las mismas están conformadas por migrantes bolivianos y ciudadanos argentinos que fueron rescatados de talleres de costura clandestinos. Frente a este contexto, el Centro se ha constituido en una incubadora social que impulsa la generación de cooperativas sustentables. Teniendo en cuenta esta finalidad, y mediante el acercamiento entre docentes y actores del CDI, en el año 2016 se realizaron encuentros de diagnóstico y acciones colectivas, siguiendo el enfoque del "aprendizaje servicio solidario" y la metodología del "conversar liberador". Esto posibilitó la generación de conocimiento y saberes para la mejora organizacional de las cooperativas y el aprendizaje colectivo entre docentes, profesionales, estudiantes y cooperativistas.

\section{Palabras clave}

- Capacidades organizacionales

- Cooperativas de trabajo

- Conversar liberador

- Mejora organizacional

- Sustentabilidad

\section{Resumo}

Na Argentina, durante as últimas décadas, foram desenvolvidas experiências universitárias de fortalecimento a incubadoras produtivas, sociais e tecnológicas. Neste sentido, o presente trabalho tem o intuito de descrever uma experiência de fortalecimento das "capacidades organizacionais" das cooperativas de trabalho que conformam o Centro Demostrativo de Indumentaria (CDI). Elas estão integradas por migrantes bolivianos e cidadãos argentinos, que foram resgatados de oficinas de costura clandestinas. Perante este quadro o Centro, constituiu-se como uma incubadora social, que impulsiona a geração de cooperativas sustentáveis. Levando em consideração esta finalidade, e por meio da aproximação entre docentes e atores do CDI, no ano de 2016, foram realizados encontros de diagnóstico e ações coletivas, seguindo a abordagem da "aprendizagem serviço solidário", e a metodologia do "conversar liberador". Isto possibilitou a geração de conhecimento e saberes para a melhora organizacional das cooperativas, e a aprendizagem coletiva entre docentes, profissionais, estudantes e cooperativistas.

Palavras-chave

- Capacidades organizacionais

- Cooperativas de trabalho

- Conversar liberador

- Melhora organizacional

- Sustentabilidade
Para citación de este artículo Chosco Díaz, G. y Camblong, J. (2017).

Fortalecimiento de las capacidades organizacionales mediante el conversar liberador. Revista +E versión en línea, 7(7), 270-279. Santa Fe, Argentina: Ediciones UNL. 


\section{Presentación general}

El presente artículo tiene por objetivo describir una experiencia de fortalecimiento de las "capacidades organizacionales" (Ackoff, 1995; Oszlak y Orellana, 2000, Schlemenson, 1990) de las cooperativas de trabajo que integran el Centro Demostrativo de Indumentaria (CDI). Este tipo de incubadora social emergió en Argentina como respuesta a las necesidades de generar condiciones laborales dignas para quienes eran rescatados de talleres de costura clandestinos.

Paulatinamente, este centro ubicado en el barrio de Barracas se fue constituyendo en un espacio de aprendizaje y de prestación de servicios ciudadanos mediante la acción congruente de diversos actores. En la actualidad, representa una novedosa organización que opera en el mercado textil, brinda servicios sociales, oportunidades laborales dignas y calidad para competir en el mercado.

Ahora bien, en el año 2015, mediante el acercamiento de docentes del Instituto de Industria de la Universidad Nacional de General Sarmiento y profesionales del Instituto Nacional de Tecnología Industrial (INTI), se fueron evaluando posibles alternativas de acción para contribuir en el fortalecimiento de la autogestión y la sustentabilidad de cada cooperativa. En esta dirección, se propuso una metodología particular para realizar las actividades en el CDI. Primero se llevó a cabo un diagnóstico a través de entrevistas grupales a los cooperativistas y a los profesionales del INTI. Segundo, se implementaron conversatorios y encuentros de intercambio de saberes.

La experiencia realizada estuvo basada en el enfoque "aprendizaje-servicio" (Tapia, 2008), una forma de aprendizaje activo y significativo, situado en el contexto de una comunidad que se convierte en lugar de participación y también en lugar de aprendizaje. Este enfoque es superador de los esquemas de intervención tradicionales, ya que confluyen dos tipos de experiencias educativas que generalmente se desarrollan aisladamente. Por un lado, actividades en función de un aprendizaje interdisciplinario y, por otro, actividades solidarias de una causa de la propia comunidad u organización. Por lo tanto, entendemos que existe un aprendizaje-servicio cuando se genera la intersección entre la intencionalidad pedagógica y solidaria. Por supuesto, esto posibilitó otras maneras de generar conocimiento sobre las cooperativas a partir de reinterpretar las teorías, a la luz de las experiencias y vivencias de referentes.

Así también, para el desarrollo de los encuentros, los orientadores siguieron la metodología del "conversar liberador"
(Maturana, 1997; Maturana y Dávila, 2006), donde se pone en movimiento la dinámica relacional reflexiva entre las personas, opera de manera inconsciente, hace emerger y fluir diversos problemas y conflictos ocultos.

Ambos enfoques resultan ser complementarios y apuntan a la generación de nuevas modalidades de acercamiento y creación de conocimiento entre cooperativistas, docentes, estudiantes y profesionales.

Con relación a la estructura del artículo, en primer lugar se realiza una breve descripción de los antecedentes de las incubadoras y se propone un modelo conceptual para el abordaje analítico del CDI. En segundo lugar, se presenta el diagnóstico efectuado de las cooperativas y del Centro, y las propuestas de acción colectivas que fueron implementadas. Por último, se expone la reflexión sobre los alcances de la experiencia y sobre las propuestas de acción territorial que se promueven a nivel gubernamental para el desarrollo de incubadoras de cooperativas de trabajo orientadas a la industria textil.

\section{Antecedentes de las incubadoras}

Las incubadoras surgen en Europa y Estados Unidos a partir de los años 50 y toman impulso en la década de los '70. Las mismas fueron pensadas como instituciones orientadas a facilitar el desarrollo de las nuevas unidades productivas mediante gestión en la búsqueda de recursos financieros, la generación de contactos comerciales y la asesoría integral.

En Latinoamérica, las primeras incubadoras comenzaron a desarrollarse en la década de los '80 como instrumentos para dinamizar la economía de los países y fomentar la creación de empleos. Se iniciaron en Brasil, a diferencia de Argentina, que empezaron una década más tarde. Diversas investigaciones (Bollati, 2015; Logegaray, 2003; Versino, 2000) coinciden en que fueron introducidas en el marco del Proyecto Columbus del Foro de Rectores de Universidades Europeas, que tuvo como objetivos promover la creación de empresas de base tecnológica desde las universidades a través de la implementación de incubadoras de empresas para crear, posteriormente, una red latinoamericana de incubadoras universitarias.

Asimismo, se asocia el inicio de las incubadoras con las actividades desarrolladas en el vivero de empresas Fray Luis Beltrán, en la Municipalidad de General San Martín, de la provincia
1) La experiencia se desarrolló en el marco del programa de Cooperativismo y Economía Social en la Universidad, de la Secretaría de Políticas Universitarias. Asimismo, guarda relación con el proyecto de investigación "De empresas recuperadas a cooperativas de trabajo. Caracterización de la transición organizacional", integrado por Claudio Fardelli Corropolese y Cecilia Chosco Díaz. 
de Buenos Aires, que actualmente tiene un programa conjunto de incubación de empresas de base social con la Universidad Nacional de San Martín y una ONG italiana. Entre los años 1995 y 2005 se fueron diseñando diversos programas estatales que promovieron la creación e instalación de incubadoras en universidades nacionales. $Y$ esto fue acompañado por la creación de organismos científicos y tecnológicos.

Estas políticas públicas de transferencia de tecnología, que tenían como foco a la universidad pública, se iniciaron para fortalecer la creación y vinculación con las empresas (Estebanez y Korsunsky, 2004). Pero con el correr de los años y de las necesidades territoriales la universidad empezó a involucrarse en otro tipo de actividades enfocadas a la transferencia universitaria solidaria con proyectos y demandas dirigidas desde diversos sectores sociales, en especial, para los más vulnerables en términos de su inserción socioeconómica y laboral (Llomovatte, Juarros, Naidorf y Guelman, 2006). En este sentido, Domecq (2005) plantea que hacia los '90 se observa la necesidad de un tipo de universidad que, si bien debe conservar ciertos componentes anteriores necesarios para su funcionamiento, tiene que incorporar o mejorar otros: una interacción aún mayor con el medio, capaz de responder a las demandas sociales y ser, además, competente para procesar las nuevas realidades que vive nuestro país.

Ahora bien, en el caso de las incubadoras no lucrativas impulsadas por la sociedad civil, centros de investigación, y entidades públicas estatales, no han enfatizado en prácticas de eficiencia y resultados sino en ampliar las oportunidades de empleo, más aún en épocas de crisis. Promediando fines de la década del '90 aparecen las incubadoras de base tecnológica y sociales vinculadas con alguna universidad $u$ organismo de la sociedad civil que se encuentra especialmente interesado en generar desarrollo en alguna área específica de conocimiento (Bollati, 2015; Logegaray, 2003). Al respecto, es inexistente la evidencia empírica y teórica de las incubadoras o centros no lucrativos; debido a ello, a continuación proponemos una estrategia conceptual de abordaje para el análisis del CDI.

El Centro es un ente público autárquico, sin fines de lucro, fue pensando como una solución para erradicar los talleres de costura clandestinos. Aunque atraviesa su décimo ciclo, aún no ha logrado consolidarse por varias razones. Una de ellas es la naturaleza de los emprendimientos, ya que se originan por la asociación de los trabajadores que son "rescatados" de las condiciones de esclavitud, ${ }^{2}$ a veces voluntaria y otras no. El desafío mayor es crear el compromiso entre ellos. Esto se suma a la difícil tarea que representa orientar a las personas para constituirse en cooperativas de trabajo cuando solo conocieron condiciones de encierro forzado como relación de dependencia. Incluso resulta complejo cuando son personas que no finalizaron los estudios primarios y deben gestionar su propia cooperativa (aprender de la gestión de la producción, la comercialización de la indumentaria, cuestiones contables e impositivas, y la búsqueda de clientes, etc.). Por último, la lógica interna del Centro, en donde intervienen y operan diversos actores estatales, sociales y políticos, se torna compleja e impredecible.

Por lo antes señalado, es necesario observar al Centro como un lugar gobernado por diversas lógicas políticas, sociales, de productividad e identidad, gestionadas de manera compartida debido a que profesionales de INTI, la Fundación La Alameda y los cooperativistas, se reúnen para prestar servicios ciudadanos. Entre estas acciones estatales y no estatales se evidencia la construcción de una gestión compartida (Poggiese y Redín, 1997) que se expresa en redes de acción territorial.

En términos teóricos, estas redes pasan a formar parte de la "paraadministración" (Ramió, 2000) o bien son considerados Organismos "ad hoc o paraestatales" (Oszlak, 1984), que no se encuentran formalmente incorporados en la estructura de la administración pública y su objetivo es resolver demandas críticas que, supuestamente, las unidades preexistentes no están en condiciones de afrontar. Asimismo, el modelo de gestión del Centro se inscribe en la propuesta de Brugué y Gomá (1994). Estos autores identifican la existencia de sistemas complejos e innovadores que enfrentan el reto de encontrar continuamente vías de impulso hacia el cambio que les exige el territorio. Estas prácticas organizacionales siguen los principios de diversidad, cambio y gestión flexible.

El principio de diversidad abre la posibilidad de atacar problemas con estrategias emergentes, capaces de responder a las situaciones de incertidumbre; el principio de cambio hace referencia a las principales características del contexto como lo son la turbulencia y la incertidumbre. Las necesidades y las soluciones cambian rápidamente, y la organización debe ser capaz de asimilarlo mejorando su capacidad de aprendizaje y
2) Dado que la temática excede los objetivos del trabajo, sugerimos la lectura Barattini (2010), donde se analizan de manera crítica las actividades ilegales de la industria textil, que utilizan mano de obra esclava de migrantes. 
adaptación; el principio de gestión flexible hace a la intervención sobre cuestiones problematizadas a partir de la participación articulada de distintos actores.

Entonces, la creación de lugares públicos co-construidos, cristalizados en sistemas decisorios dinámicos y complejos, cuyo funcionamiento depende de la postura adoptada por los actores estatales y no estatales que lo constituyen. Esto significa que todos ellos asumen una responsabilidad compartida, cada uno brinda recursos (técnicos, económicos, laborales, políticos) que estén a su alcance a fin de cumplir la misión de proveer servicios a la ciudadanía. Para finalizar, estas alianzas estratégicas lograrían conformar espacios de participación y negociación donde la idea principal descansa en la viabilización de estrategias para el desarrollo de la sociedad civil, en especial, de aquellas personas imposibilitadas o restringidas de disponer de su propia vida y sus opciones laborales.

\section{Diagnóstico de las capacidades y propuestas de acción} Para la realización del diagnóstico se tuvieron en cuenta diversas capacidades organizacionales representadas en un conjunto de aptitudes, hábitos, atributos y prácticas para la gestión de las organizaciones (Ackoff, 1995; Oszlak y Orellana, 2000, Schlemenson, 1990).

Básicamente, se identificaron aspectos organizacionales atinentes a: 1) Las relaciones sociales: competencias colectivas desarrolladas entre agentes económicos y políticos que prestan su colaboración en la provisión de información o servicios. 2) La estructura organizacional interna y distribución de funciones: funcionamiento de la unidad organizativa y capacidad funcional y física. 3) Aspectos culturales y de promoción de "reglas de juego": aquellos mecanismos de coordinación y vinculación cristalizados en acuerdos tácitos y explícitos con sus asociados y demás agentes. 4) La gestión de los recursos humanos y sus competencias: fortalecimiento del desarrollo de las capacidades individuales de los participantes en las agencias y de su involucramiento con la misión y visión de la organización. 5) El planeamiento organizacional: uno de los procesos centrales dentro de la dirección de una organización, a través del mismo se establecen los objetivos y se fijan las formas para obtenerlos, basando las acciones en cursos de acción factibles.

En tanto, lo que refiere a la organización sustentable (Etkin, 2007) entendemos que se basa en el reconocimiento de sus integrantes y en la promoción del espacio democrático, donde todos los grupos participen directa e indirectamente en la toma de decisiones. Por ello, el modelo cooperativo presenta ventajas comparativas ya que tiende a superar desigualdades, avanza en la perspectiva de la mutualidad, el esfuerzo compartido y la visión solidaria de las relaciones laborales.

Siguiendo los autores de referencia y los conceptos, se realizaron listados de preguntas para realizar las entrevistas individuales a los profesionales del Centro y las entrevistas grupales a los cooperativistas.

En el siguiente Cuadro $\mathrm{N}^{\circ} 1$ se expresan dimensiones y preguntas: 
Cuadro $\mathrm{N}^{\circ}$ 1: Dimensiones y preguntas de las entrevistas a profesionales y cooperativistas

Misión y visión

Gestión de la comercialización
¿Cuándo nace la cooperativa? ¿Quiénes participan y cómo? ¿Cuál es la misión? ¿Cuál es la visión? ¿Qué valores culturales tiene y construye?

¿Cuáles son los principales objetivos cualitativos y cuantitativos?

¿Analiza qué productos va a comercializar?

¿Conoce el mercado en el cual opera?

¿Concibe estrategias comerciales?

¿Renueva sus productos?

¿Utiliza circuitos de recepción y seguimiento de pedidos?

¿Utiliza algún método para publicidad de sus productos

¿Vende en locales propios?

¿Tiene una marca propia de sus productos?

¿Tiene métodos para estimar precios de sus productos?

¿Conoce la rentabilidad de utilizar internet como punto de venta?

\section{Decisiones}

¿Se hacen asambleas? ¿Cómo ingresan los socios?

¿Se comunican las decisiones? ¿Se comunican las acciones? ¿Cuáles son los valores cooperativos? ¿Hay un registro de las decisiones y acciones?

¿Existe un organigrama que establezca los niveles y las responsabilidades?

¿Existe división del trabajo? ¿Existen rutinas de trabajo?

¿Hay registro de las funciones de cada uno? ¿Hay trabajo en equipo? ¿Se capacita para cada puesto de trabajo? ¿Se comparten los saberes? ¿Cuenta con fuentes de información de todas las áreas? ¿Se diseñan circuitos o rutinas administrativas?

\section{Gestión de la producción y de las instalaciones}

¿Lleva registros de los stocks de insumos?

¿Lleva registros de los stocks de productos?

¿Se realizan controles del proceso productivo?

¿Existen normas de higiene y seguridad en el trabajo?

¿Controla la calidad de los insumos adquiridos?

¿Cuenta con registros de los insumos y productos que se hallan en depósito?

¿Lleva control de productos fallados?

¿Detecta riesgos en la producción?

¿Usa mecanismos de protección mientras produce?

¿Cuentan con algún método para determinar la causa de demoras en la entrega de los pedidos?

¿Cuenta con algún método de prevención de daños en el embalaje?

¿Cuenta con algún método de transporte de los productos?

¿Hay algún sistema para mantener el orden y la limpieza? ¿Realiza mantenimiento preventivo de los equipos? ¿Cuenta con algún método para determinar la causa de demoras en la entrega de pedidos?

¿Utiliza algún sistema de información contable?

¿Realiza estimaciones de volúmenes anuales de ventas?

¿Considera estimaciones de gastos y costos anuales?

¿Estima cuáles han de ser sus necesidades financieras

¿Estima incrementos en costos de producción?

¿Considera cuál es el monto mensual de efectivo necesario para su operatoria?

¿Calcula cuál es el promedio mensual de pagos?

¿Estima en promedio, a cuánto ascienden sus cobranzas mensuales?

¿Realiza estimaciones de déficit de efectivo?

¿Piensa opciones para cubrir el déficit de efectivo previsto? ¿Considera los costos financieros

originados en déficit de efectivo? ¿Se utiliza algún software?

Fuente: elaboración propia. 
a) Análisis del sistema de gestión compartida

El Centro forma parte del programa de apoyo a emprendimientos por parte del Estado. Nace de los esfuerzos conjuntos entre el Ministerio de Desarrollo Social de la Nación (aportó maquinarias y capital de trabajo para los emprendimientos); el Ministerio de Producción de la Ciudad de Buenos Aires y la Corporación Buenos Aires Sur (compraron y acondicionaron el inmueble); el Banco Credicoop y el Instituto Movilizador de Fondos Cooperativos, y la Fundación La Alameda.

Está ubicado en el barrio de Barracas. Queda a 15 minutos con el colectivo 59, aproximadamente, de la estación Constitución. En plena zona industrial, cuenta con un edificio con capacidad para 120 personas. Se encuentra acondicionado y tiene salas independientes para el funcionamiento de las cooperativas, a las cuales se les proveen las máquinas, mesas de corte compartidas, baños y comedor. Varias de las maquinarias son las secuestradas en allanamientos a talleres clandestinos.

El Centro tiene un coordinador general, una asesora en diseño de indumentaria, un comunicador social, un administrador y contador, y técnicos para las instalaciones. También un cocinero. Tienen entre 35 y 44 años. Entre sus funciones se destacan brindar acompañamiento y facilitar capacitaciones, como la generación de contactos con clientes (de primeras marcas o no), la confección de una factura, la generación del monotributo, charlas motivadoras, el diseño de prendas, etc. Entre algunos de los ejemplos de lo que se brinda podemos destacar la colaboración en la elaboración de presupuestos para clientes grandes, como también la negociación de los precios.

El horario que establece la administración es desde las 8 hasta las 17 horas. No está permitida la permanencia en el lugar fuera de ese horario por cuestiones de seguridad. Todos los gastos de servicios de luz y almuerzos son subsidiados por el gobierno de la Ciudad de Buenos Aires. La utilización de las instalaciones del centro es gratuita, no hay un tiempo predeterminado para su ocupación.

Las cooperativas de trabajo que se incuban están conformadas por jóvenes y adultos, mujeres y hombres de entre 25 y 50 años, algunos de ellos migrantes bolivianos y otros ciudadanos argentinos. Son personas adultas que no han finalizado sus estudios secundarios o bien tienen inconclusos los estudios primarios. En general, quienes deciden constituirse en cooperativas suelen ser conocidos o familiares. También se contempla la posibilidad de incorporar a nuevos integrantes que necesiten trabajo y, en este caso, deben pasar una prueba de tres meses a fin de comprobar su adaptación. Esta condición está estrictamente relacionada con el oficio de los costureros y la cultura del trabajo de los migrantes. Entre ellos comparten saberes y se capacitan para manejar una máquina o para coser las prendas, pero a veces no confían en quienes vienen a pedir trabajo. Por eso, para ser definitivamente integrante de la cooperativa debe pasar un tiempo prudencial.

Actualmente, son 6 cooperativas las incubadas. Tienen entre 6 y 20 personas. Ofrecen el servicio de confección de indumentaria para clientes que les traen las prendas cortadas y también las vienen a buscar. No producen líneas propias de productos, no tienen marcas (solo una empresa recuperada que se encuentra en la incubadora). Indistintamente se dedican a la producción por temporadas de pantalones jeans, remeras, camperas, pilotos, pantuflas, etc. La modalidad legal que les permite funcionar es el monotributo.

Habitualmente, el funcionamiento de las cooperativas se establece por decisiones colectivas. Cada integrante tiene un papel, una función, es decir, hacia el interior existe división del trabajo. Los retiros económicos son semanales, como las reglas internas.

Entre los cooperativistas y los profesionales del INTI cuidan el lugar, la separación de residuos, y el mantenimiento general. En el espacio del comedor, almuerzan todos.

Por último, cabe señalar que algunas cooperativas están ligadas a la fundación La Alameda, de la cual reciben apoyo y acompañamiento.

\section{b) Evaluación general}

En la evaluación de las cooperativas se detectaron diversos problemas, carencias y debilidades en los sistemas administrativos (procesos, técnicas, aplicaciones) que, en términos de Gilli, se definen como "el conjunto integrado de los procedimientos necesarios para concretar en actividades los objetivos de una empresa y además generar información para el control de los resultados alcanzados" (2016:173).

Al respecto, a continuación, se señalan aspectos relacionados con: - La planificación de la producción.

- Los registros formales de compras y stocks de insumos y productos.

- La comercialización y la comunicación (interna y externa).

- El presentismo, los horarios y el compromiso en el trabajo.

- El registro de las ventas y los sueldos (retiros)

de los cooperativistas.

- Los valores cooperativos y el desarrollo de una cooperativa.

- La formalización de la estructura de la cooperativa.

- El orden del taller, la limpieza, y la seguridad laboral.

- La contabilidad y la realización de documentos comerciales (cheques, facturas, remitos, presupuestos, etc.). 
Un aspecto llamativo que se detectó fue el bajo nivel de compromiso por parte de los cooperativistas y el alto nivel de ausentismo. Pareciera que tener solucionados el espacio, la maquinaria y los impuestos, interfiere en los sus emprendimientos y en el aprendizaje de la autogestión sostenible.

En cuanto al Centro, se identificaron dos aspectos, uno relacionado con el sistema de comunicación (interna, externa) que se advirtió en la despersonalización de las relaciones y, por ende, en la falta de confianza entre ellos, y la ausencia de difusión del Centro en la comunidad.

Otro aspecto fue el vinculado al Centro como incubadora y espacio de aprendizaje. Básicamente, no se registraron procesos formales de incubación y hubo una débil promoción de las habilidades de gestión social.

\section{c) Propuestas}

A los cooperativistas se les propuso avanzar en una serie de talleres y encuentros que consistirían en conversatorios grupales a fin de que ellos mismos encontraran soluciones a sus problemas. Entonces, de acuerdo con la evaluación realizada, se diagramaron los encuentros teniendo en cuenta las siguientes temáticas:

- Estrategias de comercialización e incorporación de herramientas informáticas y de la web.

- Sistema de producción y de las normas de seguridad e higiene laboral.

- Sistema administrativo y de documentos contables.

- Sistema de comunicación interna y externa.

- Identidad cooperativa.

- Impacto ambiental y territorial de las actividades textiles.

El equipo se conformó con docentes, orientadores y una estudiante voluntaria de la Licenciatura en Ecología. Se privilegió como valor fundamental la interdisciplinariedad, por eso los integrantes provenían de diversos campos de conocimiento: la administración, la antropología social, la ingeniería industrial, la informática, la comercialización, la seguridad e higiene laboral, la ecología, la contabilidad y, por último, el estudio de las letras (para el análisis discursivo, la escritura y la comunicación escrita y oral en las organizaciones).

En el caso del Centro, se les propuso a los profesionales del INTI participar en una nueva convocatoria de la Secretaría de Políticas Universitarias avanzar en una segunda experiencia, donde se abordaran conjuntamente cuestiones estratégicas de la incubadora.

\section{Aprender en la conversación con el otro: la puesta en práctica de la "Comisión de resolución de conflictos"}

¿Cómo abordar los encuentros? Conversando entre todos. De este modo, mediante disparadores conceptuales se fueron estableciendo diálogos entre los participantes a fin de que ellos mismos identifiquen situaciones críticas y posibles soluciones. A los fines de lograr una pedagogía distinta a la convencional, se recurrió al enfoque de la biología cultural, donde referentes tales como Ximena Dávila y Humberto Maturana (2006) interpretan en el "conversar liberador", una metodología basada en la reflexión y en el aprender a escuchar al otro. No es una terapia sino que se busca mediante reflexiones, preguntas y respuestas que ese otro encuentre el bienestar. De aquí se desprende que a través del conversar liberador la persona se va dando cuenta - escuchándose a sí misma- de encontrar respuestas a sus interrogantes. Esto significa que escuchar es tan importante como el mismo conversar y que lo fundamental son las ganas que tenga la persona en realizar ambas acciones, pues si no existe la intención de hacerlo será muy difícil lograr algo. Por lo tanto, la primera invitación es a conversar, luego el espacio de confianza se irá dando solo. Por eso se denomina también aprendizaje en la conversación. Entonces, mediante la conversación se evidenciaron varios problemas que venían sosteniendo entre las cooperativas. $\mathrm{Y}$, al respecto, en uno de los encuentros se decidió crear una Comisión de resolución de conflictos.

Entendemos que en el ámbito de las relaciones humanas el conflicto suele estar presente con cierta frecuencia y las causas de su aparición pueden ser de las más variadas. Hablar de conflictos no es sencillo, y menos aún en reuniones colectivas; sin embargo, el tema emergió en el taller de sistemas de producción y seguridad e higiene. Conversando con los cooperativistas de cada cooperativa, se evidenció que se conocían muy poco entre ellos y, por lo tanto, los problemas recurrentes de la sede no tenían resolución debido a la falta de compromiso general. Por ejemplo, cuestiones relacionadas con los matafuegos, con la administración de los espacios de uso común, con el mantenimiento del lugar en general. Asimismo, hacia el interior de cada cooperativa también admitieron tener conflictos entre compañeros, situaciones recurrentes vinculadas al cumplimiento de los horarios, la asistencia, la falta de tiempo para reuniones, entre otros temas.

Seguidamente, se presentan dos herramientas orientadas a la resolución de conflictos utilizadas en los conversatorios. Ambas derivan del campo de la ingeniería y fueron acuñadas en la posguerra por quienes iniciaron los conceptos de calidad, que en la actualidad encontramos en gran parte de las empresas. Por su versatilidad y amplitud, estas herramientas han dado respuesta a distintos campos con resultados excelentes. Según Lederach (1989), el conflicto tiene una estructura compuesta por tres partes: las personas, el proceso, el problema. Las herramientas aquí trabajadas justamente son de aplicación directa para la resolución de problemas y sus procesos.

Una premisa de Edward Deming es que los problemas generalmente se resuelven en parte, es decir que los problemas complejos, con multiplicidad de causas, son resueltos en etapas. El ciclo que se cumple y permite disminuir la intensidad del 
problema se denomina "Ciclo de Deming o PDCA", Planing, Do, Control, Active, lo que, traducido, significa Planificar, Realizar, Controlar, Activar.

A continuación se realiza una adaptación de la propuesta de Deming en conjunción con otra herramienta propuesta por la Organización Internacional del Trabajo a la hora de encontrar solución a problemas de procesos.

En principio, ante un problema complejo, lo principal no es actuar, la propuesta que se propone indica realizar un plan $(\mathrm{P})$, y ese plan incluye:

- Primera instancia: a) entender el problema, b) planear objetivos.

- Segunda instancia: a) buscar las causas, b), encontrar las causas raíces y encaminar la solución, c) idear un plan para resolver.

Luego se debe poner en marcha el Plan, (D), aquí es donde realmente comienza la solución del problema y debemos empezar a Controlar (C).

En tanto, Deming propone Activar como actividad donde buscaremos que el conflicto no vuelva a suceder.

El proceso detallado de cada una de las etapas es el siguiente: En la primera etapa del Plan, el ítem a), para entender el problema, en esta adaptación proponemos utilizar la técnica del interrogatorio ${ }^{3}$ en su etapa preliminar. Aquí las preguntas a realizar a los actores del conflicto son: ¿Qué es lo que pasa? ¿Por qué pasa? ¿Dónde pasa? ¿Cuándo pasa? ¿Cómo pasa? Una vez finalizada esta actividad se pasa a la siguiente, para ello se debe recordar las características de los objetivos. Dichas características son:

- Enfocado, característica del objetivo que indica que el tema tiene incumbencia con el problema a atender.

- Desafiante, indica que debe requerir un esfuerzo.

- Realista, un objetivo debe poder cumplirse con los recursos con los que cuenta la empresa.

- Cuantificables, refiere a que siempre debe incluir a una cantidad determinada.

- Acotados en tiempo y espacio, característica que indica que el objetivo debe tener un tiempo para su cumplimiento y un determinado lugar de acción.

En tal caso, es necesario colocar un objetivo consensuado entre los involucrados, debiéndose tener en cuenta que el mismo no es necesario que solucione totalmente el problema ya que un nuevo ciclo, como se dijo, podrá mejorar en un segundo nivel el conflicto.
Un ejemplo dado fue el siguiente: "Se pretende disminuir en un $60 \%$ las discusiones entre los compañeros Pablo y Juan para la próxima quincena en la oficina que comparten".

Para la segunda instancia del Plan, se pretenden encontrar la mayor cantidad de causas que generan el problema. Del cuestionario preliminar, seguramente han surgido una cantidad importante de causas, especialmente para el caso de la pregunta a) 1 y a) 2 si la respuesta es tiene muchas componentes, es conveniente realizar un análisis de lo que se denomina búsqueda de la causa raíz. Para ello, otras dos herramientas puedan dar muy buenos resultados, denominadas espina de pescado ${ }^{4}$ y cinco porque, ${ }^{5}$ ambas tienen la misma filosofía, y es preguntar ¿por qué?, hasta que la respuesta sea porque sí, es decir, ella es la raíz. Otro ejemplo dado de un caso típico de conflicto: "Usa mi escritorio", "lo escucho hablar todo el día y no cumple", "trae perfume fuerte y me da alergia", "no llega a horario", "escucha música mientras trabajo" y más...”.

Al buscar la causa raíz de cada una de estas causas podemos encontrar que esta (la raíz) es el escaso lugar en la oficina y operando sobre este se solucionan todas las anteriores. Una muy buena opción para encontrar soluciones para las causas raíces es la aplicación del cuestionario profundo de la técnica del interrogatorio. Este busca que los actores (quienes conocen las verdaderas causas del conflicto) propongan soluciones, y toma esta forma:

1. ¿Qué debería pasar?

2. ¿Dónde debería estar?

3. ¿Cómo debería comportarse?

4. ¿En qué momento debería suceder?

Y a cada respuesta se le debe agregar: ¿y qué propones vos? Seguramente, luego de un interrogatorio profundo, y con la respuesta a la pregunta final ¿y que propones vos?, aparecerán claras ideas de solución. Quedará luego instrumentar los medios, adaptar las soluciones, conseguir los recursos, o lo que sea necesario para brindar la solución propuesta o aproximadamente propuesta por los cooperativistas.

A medida que cada una de las causas raíces se soluciona debemos controlar que el conflicto vaya disminuyendo; y después de dar una solución a cada una de las causas raíces el conflicto habrá finalizado. Para el caso en que el conflicto continúe (seguramente con menor intensidad) deberemos repetir el ciclo hasta eliminar el problema.
3) Técnica del interrogatorio propuesta de la OIT para entender un problema por parte de aquellos que lo sufren a diario. Esta técnica se compone de dos etapas, la preliminar (donde nos enteramos del problema) y la profunda (donde buscamos una solución al problema).
4) Herramienta propuesta por el Ing. Ishikawa, compañero de trabajo en los círculos de calidad puestos en marcha en Japón por Deming para la búsque- da de causas raíces.

5) Herramienta propuesta por Deming para la búsqueda de causas raíces. 


\section{Reflexión final}

En Argentina, durante estas últimas décadas la investigación científica fue y es parte de un proceso político educativo en el que participan el Estado, la universidad pública nacional y agentes de la sociedad civil. De lo visto hasta aquí, se desprende que la vinculación universidad-ONG en nuestro país es un proceso complejo que aún no se ha consolidado. Consideramos que hacia el interior de las universidades nacionales los equipos docentes fueron redireccionando su interés hacia distintos agentes territoriales. Las ONG tardaron en despertar el interés académico, sin embargo en la actualidad los procesos de emergencia y transformación tanto de asociaciones civiles como fundaciones pasaron a ser de primordial interés para la investigación y la extensión.

Este artículo tuvo como principal objetivo describir la experiencia de fortalecimiento y aprendizaje de las cooperativas de trabajo del centro demostrativo de indumentaria. Los encuentros interdisciplinarios posibilitaron reunir a los cooperativistas, generar debates y diagnósticos sobre la gestión cotidiana, las dificultades y los desafíos futuros.

En el ámbito de los encuentros se propició la circularidad de los saberes y conocimientos de manera transversal y horizontal. Asimismo, se realizaron actividades que provocaran la mirada crítica de los cooperativistas sobre sí mismos, a fin de que puedan identificar las amenazas, las fortalezas y transformar las debilidades en oportunidades.

Como resultado final se generó un espacio de aprendizaje colectivo que tuvo como principales receptores a los docentes, a la estudiante que participó, y a los actores del Centro. Entre los logros de esta experiencia se destaca la apropiación del conocimiento para la práctica diaria de las cooperativas. Entre algunos temas, se encuentran las cuestiones contables, de seguridad e higiene, y de gestión sustentable (democratización de las decisiones). Igualmente, consideramos un antecedente importante la creación de la Comisión de conflictos ya que se evidenciaron problemas de gestión transversales hacia el interior de la incubadora. En parte esto posibilitó que se conocieran los cooperativistas y que compartieran preocupaciones sobre las instalaciones del Centro. En cuanto a la mirada crítica sobre la dinámica de gestión y la acción territorial del CDI, es trascendental remarcar tres aspectos. Primero, el sistema general de funcionamiento del Centro presenta amplias potencialidades que no son exploradas. La capacidad instalada está subutilizada. Actualmente transita una situación crítica el sector textil, lo cual afecta a la incubadora. Esto genera que las personas vuelvan a trabajar bajo dependencia, retornen a los talleres o bien a su país. Es decir, no se implementan estrategias para consolidar un modelo innovador que ha demostrado que supera ampliamente sus posibilidades del mercado al mismo tiempo que a las empresas tradicionales. Segundo, existe una fuerte tensión entre la lógica de trabajo de los migrantes, la racionalidad burocrática que se establece en el proceso de incubación y la lógica de La Alameda. Consideramos que esto impide el avance y consolidación de un modelo colectivo de gestión compartida. Desde el campo de los estudios organizacionales y de la administración es importante aportar y aprender, debido a que este tipo de incubadoras está transitando su fase inicial. ¿Por qué? Los procesos de incubación no son lineales, unidireccionales, planeados por un solo actor, tampoco pautados en tiempos determinados; por el contrario, tienden a crearse procesos complejos de aprendizaje colectivo en donde cada cooperativa comprenderá paulatinamente su papel como empresa cooperativa, sociales, productivas y con una identidad propia. En efecto, se impulsa la generación de una organización democrática, que posee un modo de gestión distinto de las Pymes y empresas.

Tercero, es notable la valorización del trabajo digno y la calidad de la indumentaria que se produce en el Centro, pero poco se promociona en la comunidad. Advertimos la necesidad de divulgar las actividades que allí se realizan y de ampliar el papel que tiene la incubadora en el aprendizaje de los estudiantes universitarios. El acercamiento tanto de los cooperativistas como de los profesionales generaría la circulación de saberes y el descubrimiento de muchos jóvenes en sus propias carreras. Y luego de haber explorado el modelo de incubación social de cooperativas de trabajo, creemos que representa un tipo particular de política pública de acción territorial. La misma debe trascender como una estrategia de desarrollo y equidad, por lo que, resulta necesario promocionarlas a nivel municipal y regional. Esto impactaría en la apertura de nuevas modalidades de trabajo en la economía social, y en la economía de mercado.

Finalmente, consideramos necesaria la ampliación de este tipo de experiencias de manera continua, perdurable y sistemática, para la generación de conocimientos socialmente útiles. Por supuesto, mediante la implementación de políticas públicas universitarias y programas destinados a sostener a equipos de jóvenes docentes investigadores que hayan elegido el camino de la extensión o de los servicios a la comunidad como una actividad académica que debe ser reconocida en el sistema universitario. 


\section{Referencias bibliográficas}

Ackoff, R. (1995). Planificación de la Empresa del Futuro. México: Limusa. Barattini, M. (2010). Trabajo esclavo y organización: el caso de la Unión de Trabajadores Costureros en Argentina. Estudios Demográficos y Urbanos, 461-481. Bollati, L. (2015). Análisis de los modelos de incubación existentes: caso de estudio de la Red de Incubadoras de América Latina. Tesis de Maestría. FLACSO. Sede Académica Argentina, Buenos Aires.

Brugué, Q. y Gomà, R. (1994). Nuevos modelos de gestión y organización pública. Autonomies, 2-19.

Maturana, H., y Dávila, X. P. (2006). Desde la matriz biológica de la existencia humana. PReLac, (2), 30-39.

Domecq, R. (2004). Pensar la universidad a fines de/ siglo XX. Notas sobre la creación de la Universidad de General Sarmiento. S/d.

Estebanez, M. y Korsunsky, L. (2004). Medición de actividades de vinculación y transferencia de conocimientos científicos y tecnológicos. En El Estado de la Ciencia. 2003 Principales indicadores de ciencia y tecnología Iberoamericanos/ Interamericanos. RICYT, CYTED, Redes.

Etkin, J. (2007). Capital social y valores en la organización sustentable: el deber ser, poder hacer y la voluntad creativa. Buenos Aires: Granica.

Gilli, J. J.; Arostegui, A.; Doval, I.; lesulauro, A. y Schulman, D. (2016). Diseño organizativo. Estructura y procesos. Buenos Aires: Granica.

Krick, E. (2000). Ingeniería de Métodos. 15ª ed. México: Limusa.

Llomovatte, S., Juarros, F.; Naidorf, J. y Guelman A. (2006). La vinculación universidad-empresa: miradas críticas desde la universidad pública. Buenos Aires: Mino y Dávila.

Logegaray, V. (2003). Gestión de empresas innovadoras: las incubadoras de empresas en argentina. Documentos. Publicación del Centro de Estudios de Sociología del Trabajo No 41, abril-junio.

Maturana, H. (1997). Emociones y lenguaje en educación y política. Chile: Dolmen.

Meyers, F. E. (2000). Estudios de tiempos y movimientos: para la manufactura gil. Pearson educación.
Miller, D. M. S. (1992). Ingeniería industrial e investigación de operaciones (№ 658.5 M652i). México: Limusa.

Organización Internacional del Trabajo (1992). Introducción al estudio del trabajo. $4^{\mathrm{a}}$ ed. Ginebra.

Oszlak, O. (s/f). Diagnóstico estratégico para la reforma del Estado en América Latina: orientaciones, metodologías e instrumentos. S/d.

Oszlak, O.; Orellana, D. (2000). El análisis de la capacidad institucional: aplicación de la metodología SADCI. Documento TOP sobre gestión pública. Centro de desarrollo y asistencia técnica en tecnología para las organizaciones públicas. Poggiese, H. y Redín M. E. (1997). La región Oeste de la Ciudad de Buenos Aires. La gestión asociada en la red regional. Serie de Documentos e Informes FLACSO N²20.

Prokopenko, J. (1997). La Gestión de la Productividad. Primera reimpresión. México: Limusa.

Ramió, C. (2000). Externalización de servicios públicos y corrientes neoempresariales: Ios problemas de la administración relacional en el contexto de un gobierno multinivel. CLAD.

Salvendy, G. (1991). Manual del Ingeniero Industrial. Volumen I-II. México: Limusa. Schlemenson, A. (1990). La perspectiva ética en el análisis organizacional. Buenos Aires: Paidós.

Tapia, L. (2008). Política Salvaje. La Paz: CLACSO, Coediciones La Paz CLACSO, Muela del Diablo, Comunas.

Tobelem, A. (1993). Sistema de análisis y desarrollo de la capacidad institucional (SADCl). Manual de operaciones. División de administración del sector público. Publicaciones ocasionales. LATPS Nº 9.

Versino, M. (2000). Las incubadoras universitarias de empresas en la Argentina: reflexiones a partir de algunas experiencias recientes. Redes, 7(15, agosto), $151-181$.

\section{6 \\ Los procesos de incubación no son lineales, unidireccionales, planeados por un solo actor, tampoco pautados en tiempos determinados; por el contrario, tienden a crearse procesos complejos de aprendizaje colectivo}

